\title{
Gut Amyloidosis in Lepromatous Leprosy Regressing with Therapy
}

\author{
R. R. GRIGOR, W. R. LANG and G. I. NICHOLSON \\ Departments of Gastroenterology and Infectious Diseases, Auckland Hospital, New Zealand
}

\begin{abstract}
Two Samoan patients with gut amyloidosis secondary to lepromatous leprosy were studied for five years. Intestinal "pseudo-obstruction" and malabsorption were prominent early features. Evidence suggesting regression of the amyloid deposits following control of the leprosy included clinical, biochemical, radiological and histological data.
\end{abstract}

\section{Introduction}

Lepromatous leprosy may be complicated by amyloidosis. When gastrointestinal amyloidosis occurs, malabsorption and "pseudo-obstruction" may be serious clinical features (Gilat and Spiro, 1968). Regression of amyloid in patients has only occasionally been documented before, when improvement of secondary amyloidosis has followed therapy directed at the underlying disease (Triger and Joekes, 1973).

A five year study of two patients with lepromatous leprosy complicated by amyloidosis of the gastrointestinal tract causing malabsorption and "pseudoobstruction" is reported. The follow up data suggest regression of the amyloid.

\section{Case Reports}

\section{CASE 1}

Mrs J. B. was diagnosed as having leprosy in Samoa in 1944 when aged 21. She received 12 years of chemotherapy on Makogai Island. She came to New Zealand in 1959. Lepromatous leprosy was confirmed in Auckland by skin biopsy in 1963, and because she was considered infectious she underwent six years hospitalisation. Treatment with dapsone, thiambutosine, and clofazimine was given.

Four years after admission she gradually developed weight loss, cachexia and weakness. At this time skin biopsy specimens were submitted for mouse inoculation testing and Myco. leprae resistant to dapsone were reported. Treatment with dapsone was stopped.

In 1968 a faecal fat excretion exceeding $50 \mathrm{~g}$ daily on a ward diet confirmed malabsorption. Other laboratory investigations included calcium $5.2 \mathrm{mg} / 100 \mathrm{ml}$, albumin $0.9 \mathrm{~g} / 100 \mathrm{ml}$, prothrombin clotting time less than $5 \%$, "flat" glucose tolerance curve, and $d$-xylose, and vitamin B 12 (Schillings) absorption tests at the 
lower limit of normal. Gross indicanuria was present. Amyloid deposition was found in the lamina propria of a rectal biopsy, but not in a per-oral jejunal mucosa biopsy or in a needle biopsy of the liver.

Intermittent, but increasingly frequent and severe episodes of colicky abdominal pain and post-prandial vomiting occurred. A barium series revealed grossly impaired small bowel peristalsis (Fig. 1). A laparotomy was performed, but showed no evidence of a mechanical obstruction. The stomach and duodenum felt thickened, but the large bowel, liver, spleen, pancreas and kidneys were macroscopically normal. Sirius staining of jejunal biopsy specimens demonstrated large deposits of amyloid in the lamina propria and in the walls of submucosal vessels (Fig. 2). Vessel wall deposition was also seen in the appendix and liver.

Drug malabsorption was suspected and the dose of clofazimine was increased from $600 \mathrm{mg} /$ week to $600 \mathrm{mg} /$ day. Doxycycline $200 \mathrm{mg} / \mathrm{day}$ was added for its postulated antilepromatous effect (Opromola et al., 1970) and to control the bacterial invasion of the upper small bowel which was probably causing the indicanuria. Over the next five months a marked improvement in gastrointestinal symptoms and general condition was noted. Nasal septum and skin smears became negative, enabling discharge from hospital. Within a year, faecal fat excretion was reduced to $6 \mathrm{~g} / \mathrm{day}$ and the radiological appearance of the small bowel was

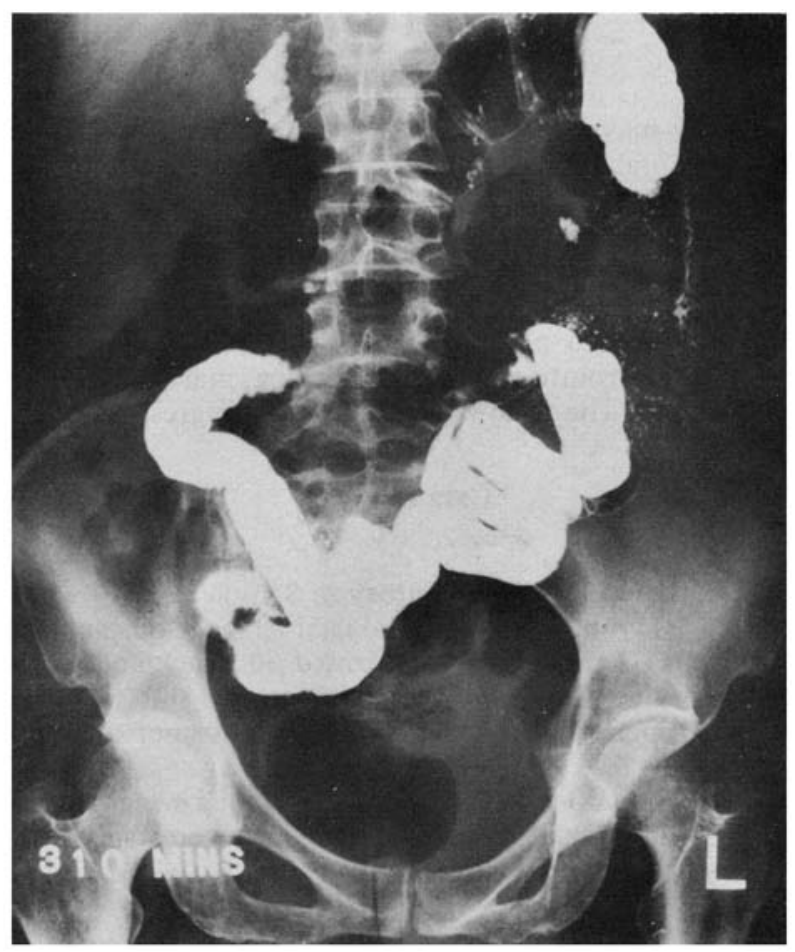

Fig.1.(Case 1.) Barium small bowel X-ray at $5 \mathrm{~h}$ shows grossly impaired small bowel peristalsis. (24 June 1968.) 


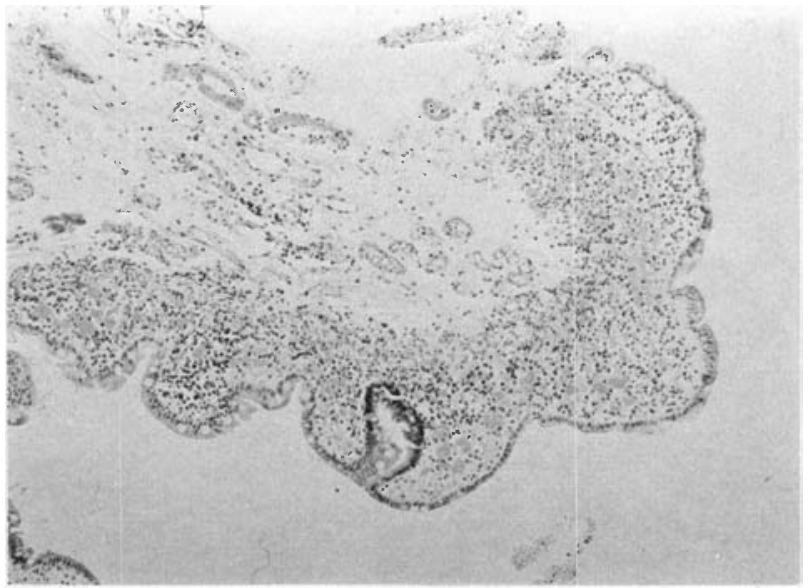

Fig.2.(Case 1.) Jejunal biopsy obtained at laparotomy. Large deposits of amyloid are shown in the lamina propria and in the walls of the submucosal vessels (sirius stain). (27 June 1968.)

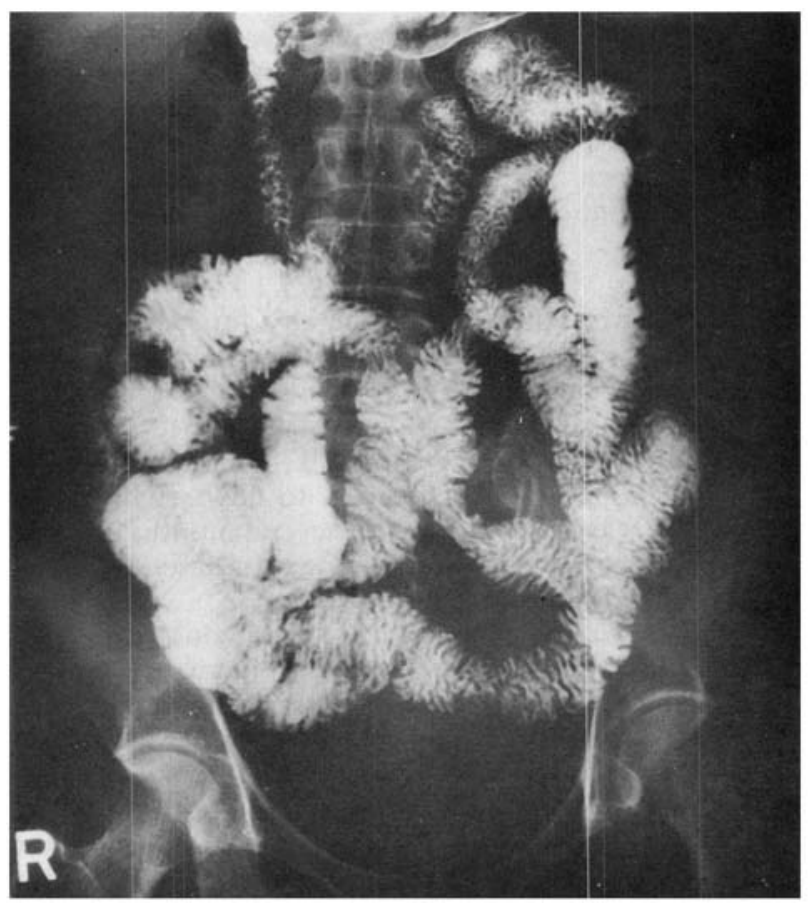

Fig.3.(Case 1.) Barium small bowel X-ray at $30 \mathrm{~min}$. Following three years of effective treatment of the leprosy, the tracing is now within normal limits. (30 April 1971.) 


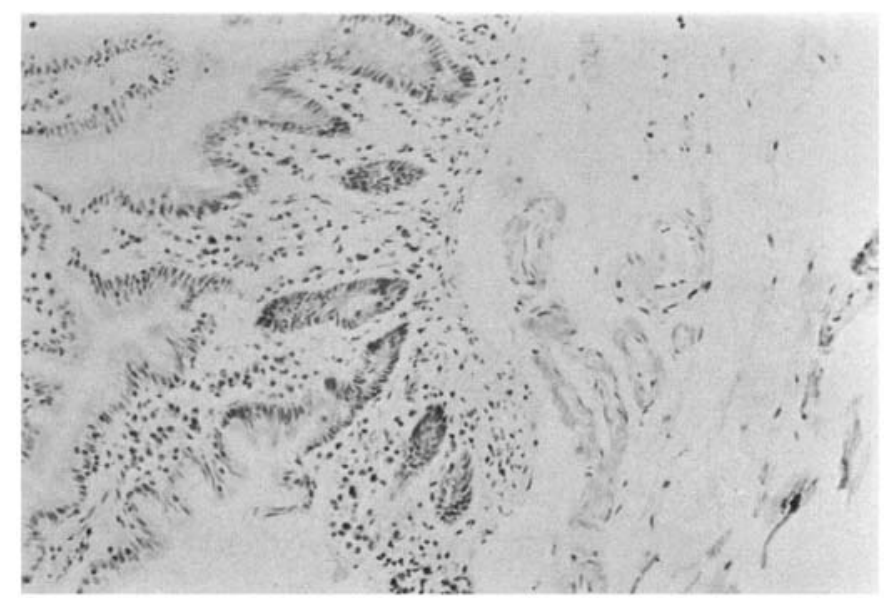

Fig.4.(Case 1.) Per-oral jejunal biopsy. The amount of amyloid deposition has decreased following three years of effective treatment of the leprosy (sirius stain). (6 May 1971.)

improved. Three years following discharge, she remained free from bowel symptoms. Faecal fat excretion and small bowel X-rays were normal (Fig. 3). A random per-oral jejunal mucosal biopsy showed a smaller amount of amyloid deposition than had been present in the surgical specimen (Fig. 4). The clofazimine has now been stopped, but she continues to take rifampicin $600 \mathrm{mg} /$ day and doxycycline $200 \mathrm{mg} /$ day.

\section{CASE 2}

$\mathrm{Mr}$ A. C. has had intermittent dapsone therapy for leprosy in Western Samoa since aged 15 years. He presented in Auckland with the cutaneous manifestations of lepromatous leprosy when aged 24, in 1968. There were minimal systemic symptoms. Dapsone and clofazimine were prescribed but positive smears containing solid staining organisms were still being obtained even after the addition of rifampicin. Some doubt existed as to how reliably he was taking his medication. In 1971 he was readmitted with several months' intermittent colicky abdominal pain and post-prandial vomiting. He also described four to five loose bowel motions/day. Weight loss had been three stone.

On examination, he was cachetic with finger clubbing and tetany. Mild hepatomegaly, slight abdominal distension and increased bowel sounds were present. There was laboratory confirmation of malabsorption; faecal fat excretion was $12 \mathrm{~g} /$ day on a ward diet, serum calcium $5.2 \mathrm{mg} / 100 \mathrm{ml}$, albumin $1.2 \mathrm{~g} / 100 \mathrm{ml}$, prothrombin clotting $37 \%$, "flat" glucose tolerance curve, and impaired $d$-xylose absorption. A plain abdominal X-ray showed fluid levels and gas in both small and large bowels (Fig. 5). A barium X-ray of the small bowel demonstrated delayed transit time, dilated loops of proximal jejunum, areas of segmental narrowing and coarsening of the mucosal folds (Fig. 6). A per-oral jejunal mucosal biopsy showed amyloid deposition in the deeper layers of the lamina propria, particularly round vessels in the submucosa (Fig. 7). Jejunal disaccharidase deficiency was also demonstrated. 


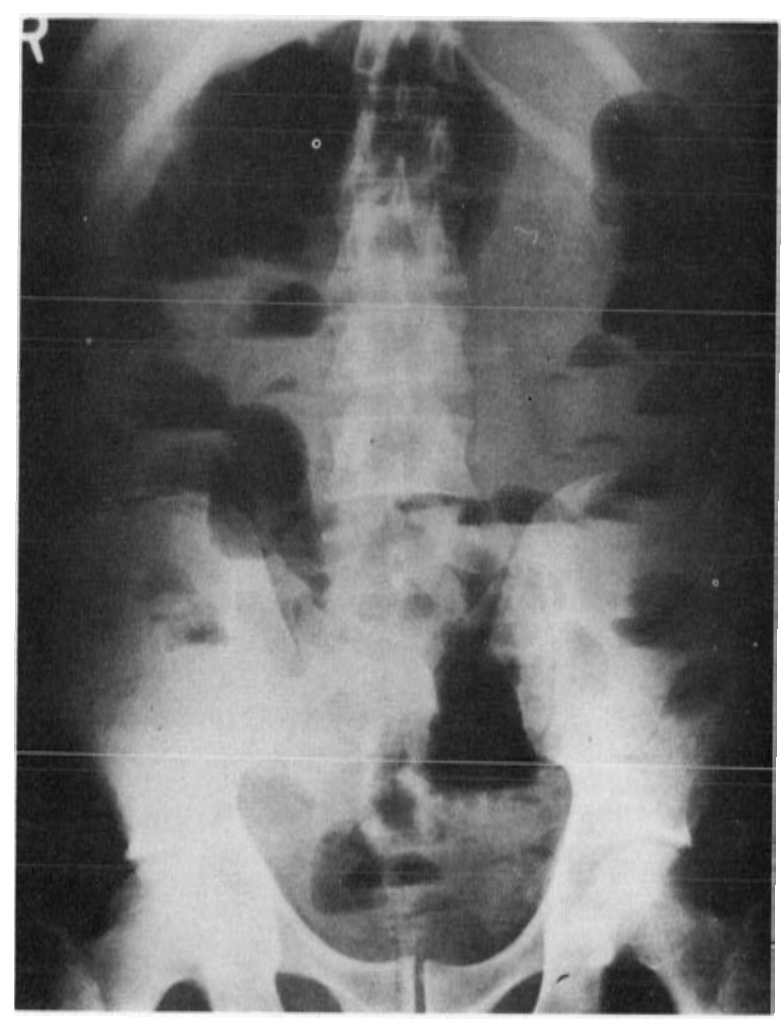

Fig.5.(Case 2.) Plain X-ray of abdomen shows fluid level and gas in both small and large bowels. Serum electrolytes were normal. (29 March 1971.)

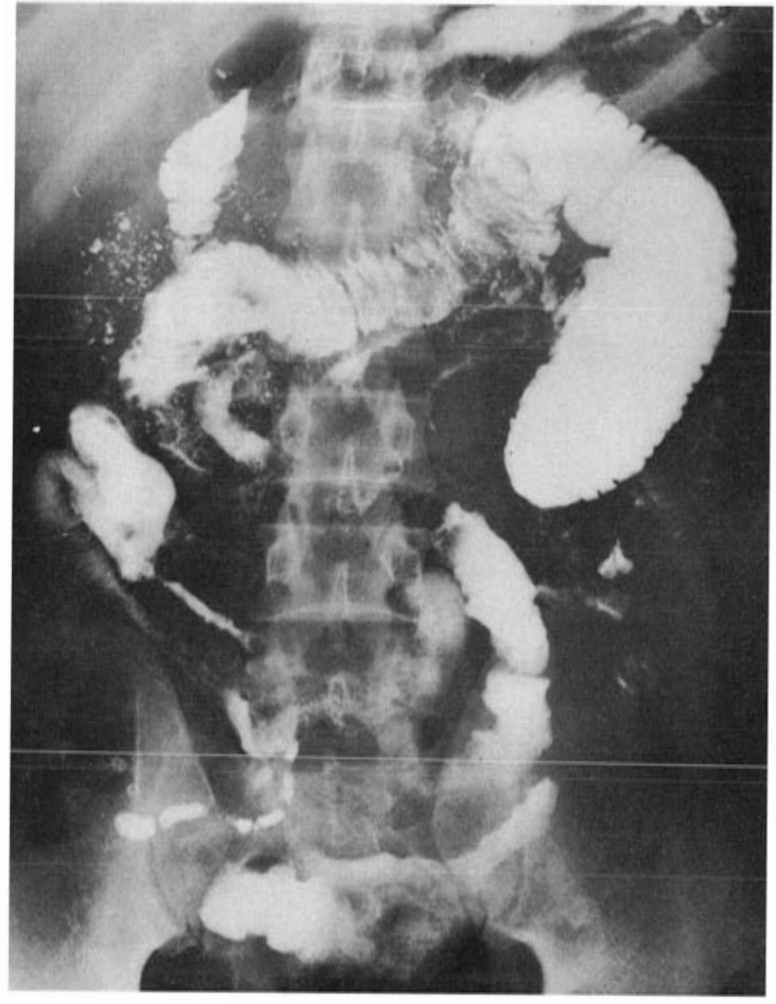

Fig.6.(Case 2.) Barium small bowel X-ray at $3 \mathrm{~h}$ demonstrates delayed transit time, dilated loops of proximal jejunum, areas of segmental narrowing, and coarsening of mucosal folds. (5 March 1971.) 


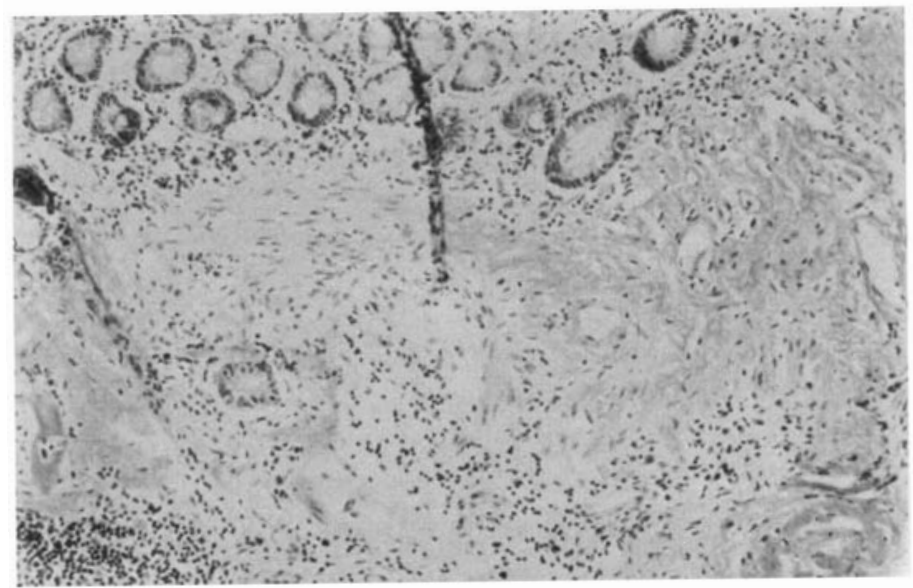

Fig.7.(Case 2.) Per-oral jejunal mucosal biopsy. Amyloid deposition in the deeper layers of the lamina propria, particularly around vessels in the submucosa (sirius stain). (8 March 1971.)

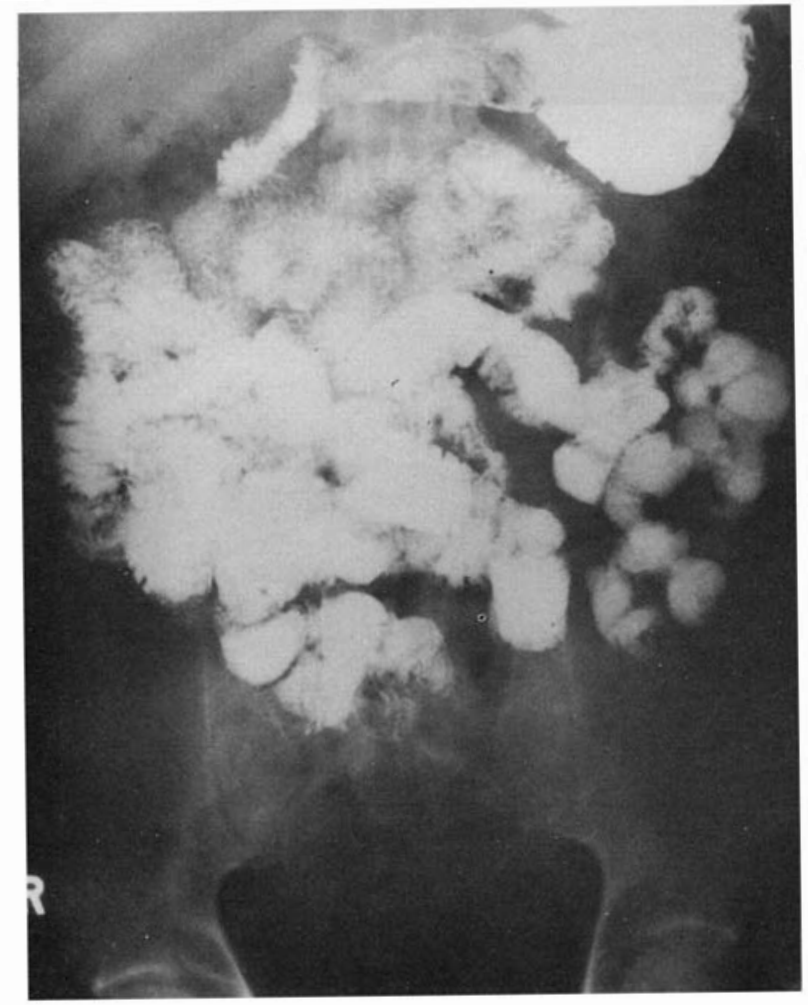

Fig.8.(Case 2.) Barium small bowel X-ray at 15 min. Following more intensive treatment of the leprosy for 18 months, the tracing is now within normal limits. (30 August 1972.) 


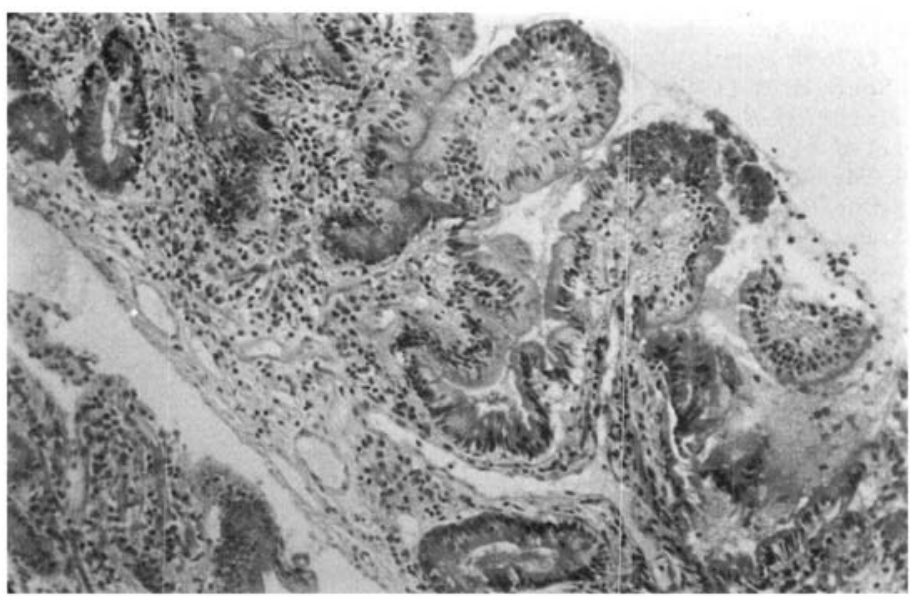

Fig.9.(Case 2.) Per-oral jejunal biopsy. The amount of anyloid deposition has decreased following 18 months effective treatment of the leprosy. (31 August 1972.)

The "pseudo-obstruction" syndrome was diagnosed and managed conservatively with intermittent intravenous therapy for two months. In view of the malabsorption, the dose of antilepromatous drugs was increased, and he was started on a "triple" regime of clofazimine $600 \mathrm{mg} /$ day, rifampicin $1200 \mathrm{mg} /$ day, and doxycycline $200 \mathrm{mg} /$ day. There was gradual clinical improvement and one year later he had no gastrointestinal symptoms and no clinical or laboratory evidence of malabsorption. Barium X-rays of the small bowel were normal (Fig. 8). A repeat per-oral jejunal biopsy showed less amyloid deposition in the bowel wall than previously (Fig. 9). He continues to take a reduced dose of clofazimine, rifampicin and doxycycline. His smears for leprosy bacilli remained negative.

\section{Discussion}

Amyloidosis is a well-described complication of lepromatous leprosy (Beddow and Tilden, 1960; Cohen, 1967; Gilat et al., 1969). Malabsorption and the "pseudo-obstruction syndrome" are recognized manifestations of amyloidosis involving the gut (Gilat and Spiro, 1968; Legge et al., 1970). The diagnosis of pseudo-obstruction and its management by conservative means is important, as surgery is hazardous in these patients who are often critically ill (Legge et al., 1970; Leading Article, British Medical Journal, 1973).

It is suggested that regression of the gut amyloidosis has occurred in these two patients. The clinical, biochemical and radiological data showed definite improvement following more effective treatment of the underlying leprosy. Although the comparison of small serial biopsies is complicated by sampling problems, there is also some histological evidence of regression.

\section{References}

Beddow, M. D. and Tilden, I. L. (1960). Malabsorption syndrome due to amyloidosis of the intestine secondary to lepromatous leprosy: Report of a case. Ann Int. Med. 53, 1017. 
Cohen, A. S. (1967). Amyloidosis. New Engl. J. Med. 277, 574.

Gilat, T. et al.(1969). Deposition of amyloid in the gastrointestinal tract. Gut 10, 98.

Gilat, T. and Spiro, H. M. (1968) Amyloidosis and the gut. Am.J. Dig. Dis. 13, 619.

Leading Article (1973). British Medical Journal.

Legge, D. A. et al. (1970). Intestinal pseudo-obstruction in systemic amyloidosis. Gut 11, 764.

Opromola, D., Mendes, J. P. and de Souza, L. (1970). Treatment of lepromatous leprosy with doxycycline. Proceedings of the International Congress of Dermatology, Tokyo.

Triger, D. R. and Joekes, A. M. (1973). Renal amyloidosis-A fourteen-year follow-up. Q. J. Med. N.S. 42, 15 . 